\title{
Expression of Mineralization Markers during Pulp Response to Biodentine and Mineral Trioxide Aggregate
}

\author{
Mariana O. Daltoé, DDS, * Francisco Wanderley G. Paula-Silva, DDS, MSc, PhD, *t \\ Lúcia H. Faccioli, PbD, ${ }^{\dagger}$ Patrícia M. Gatón-Hernández, DDS, MSc, PbD, ${ }^{\ddagger}$ \\ Andiara De Rossi, DDS, MSc, PhD, * and Léa Assed Bezerra Silva, DDS, MSc, PhD *
}

\section{Ahstract}

Introduction: The purpose of this study was to compare the cell viability of dental pulp cells treated with Biodentine (Septodont, Saint-Maur, France) and mineral trioxide aggregate (MTA) and the in vitro and in vivo expression of mineralization markers induced by the 2 materials. Methods: Human dental pulp cells isolated from 6 permanent teeth were stimulated with Biodentine and MTA extracts. Cell viability was determined using the 3-(4,5-dimethylthiazol-2-yl)2,5-diphenyl tetrazolium bromide assay, and quantitative reverse-transcriptase polymerase chain reaction was used to determine the expression of mineralization markers. Specimens of teeth from dogs treated with Biodentine and MTA after pulpotomy were used to determine the presence of osteopontin and alkaline phosphatase by immunohistochemistry and runtrelated transcription factor 2 by immunofluorescence. Results: No significant differences in cell viability were found between MTA and Biodentine extracts and controls after 24 and 48 hours $(P>.05)$. After 48 hours, osteopontin (SPP1), alkaline phosphatase $(A L P)$, and runt-related transcription factor 2 ( $R U N X 2)$ expression was higher in MTA and Biodentine than in controls $(P<.05)$. Osteopontin staining was more intense and spread over a greater number of areas in Biodentine than in MTA samples $(P<.0001)$. Alkaline phosphatase staining of a mineralized tissue bridge was significantly different between materials $(P<.0001)$, but no difference in alkaline phosphatase staining of pulp tissue was found between MTA and Biodentine $(P=.2)$. Also, no significant difference in the number of cells labeled for runt-related transcription factor 2 by immunofluorescence was observed between materials $(P>$.05). Conclusions: Biodentine stimulated similar markers as MTA, but staining was more intense and spread over a larger area of the pulp tissue. (J Endod 2016; $\square: 1-8)$

\section{Key Words}

Biodentine, dental pulp, mineralization, mineral trioxide aggregate, pulpotomy

$\mathrm{M}$ ineral trioxide aggregate (MTA) (ProRoot MTA; Dentsply Tulsa Dental, Johnson City, TN) has been extensively used in endodontics to protect pulp tissue after pulpotomy for carious and traumatic exposures. Biodentine (Septodont, Saint-Maur, France) is a novel root-end filling material, which is a powder consisting mainly of tricalcium silicate $\left(\mathrm{Ca}_{3} \mathrm{SiO}_{5}\right)$, zirconium oxide, and calcium carbonate and the mixing liquid is composed of water, calcium chloride, and a hydrosoluble polymer (1). Similar to MTA, Biodentine has been shown to release calcium hydroxide (2), induce reparative dentin synthesis (3), and have antibacterial activity (4). Biodentine offers an additional advantage over MTA in that it can be used as a temporary enamel substitute and permanent dentin substitute (5). Because this new material comes in contact with both pulp and periapical tissues, biocompatibility is crucial (6).

Biodentine has shown tissue compatibility and exhibited tissue bridge formation similar to that of MTA (7). Human clinical cases using Biodentine for conservative and radical endodontic treatments showed satisfactory results regarding the induction of mineralization (8-10). However, the differentiation of pulp cells into odontoblastlike cells $(11)$ and the mineralization $(3,12)$ induced by this material are not fully known.

Osteopontin (gene name SPP1), alkaline phosphatase (gene name $A L P L$ ), bone sialoprotein (gene name IBSP), dentin sialophosphoprotein (gene name DSPP), and dentin matrix protein 1 (gene name DMP1) have been used as markers of differentiation in osteoblasts and odontoblasts to investigate the mechanisms of mineralization (13-16). Alkaline phosphatase is found in the early stages of osteoblast and odontoblast differentiation and provides inorganic phosphate to form hydroxyapatite (ie, the first step of mineralization) (17). Osteopontin is an extracellular matrix acidic glycoprotein that has been implicated in many physiological and pathological events, including the maintenance or reconfiguration of tissue integrity during inflammatory processes (18). The runt-related transcription factor 2 (gene name RUNX2) has been linked to bone and dental mineralization and plays a primary role in the differentiation of osteoblasts and homeostasis of mineralized tissues $(19,20)$.

However, further in vivo studies with Biodentine are needed to elucidate the mechanism of action for biostimulation because differences have been reported between Biodentine and MTA. Thus, this study aimed to compare the cell viability of dental pulp cells treated with Biodentine and MTA and the expression of mineralization markers in vitro and in vivo induced by both materials.

From the *University of São Paulo, School of Dentistry of Ribeirão Preto, and ${ }^{\dagger}$ Universidade de São Paulo, Departamento de Análises Clínicas, Toxicológicas e Bromatológicas, Faculdade de Ciências Farmacêuticas de Ribeirão Preto, Ribeirão Preto, São Paulo, Brazil; and ${ }^{\ddagger}$ University of Barcelona, Barcelona, Spain.

Address requests for reprints to Léa Assed Bezerra Silva, Avenida do Café, s/n 14040-904, Ribeirão Preto, SP, Brazil. E-mail address: lea@forp.usp.br $0099-2399 / \$$ - see front matter

Copyright (C) 2016 American Association of Endodontists. http://dx.doi.org/10.1016/j.joen.2015.12.018 


\section{Explant Cell Gulture}

\section{Materials and Methods}

Teeth extracted for orthodontic reasons were obtained after informed patient consent $(n=6)$ and under institutional review board approval (process \# 25315013.0.0000.5419). Human dental pulp cells were isolated from collected teeth and grown in medium consisting of Dulbecco's Modified Eagle's medium (DMEM) supplemented with 10\% fetal bovine serum, $1 \%$ penicillin/streptomycin, and amphotericin. Cells from the third through fifth passages were used for experimentation.

\section{Preparation of Materials}

This study is in accordance with ISO 10993-5:2009 (21). The extraction method was used to put the materials (Table 1) in contact with the cells tested. Two cylindric matrices $10 \mathrm{~mm}$ in diameter and $5 \mathrm{~mm}$ in height were prepared from metallic matrix bands. The materials were mixed according to the manufacturer's instructions on a sterile glass slab in a laminar flow cabinet and introduced into the sterile matrices using sterile instruments for a volume of $47.5 \mathrm{~mm}^{3}$.

The material prepared was kept in the laminar flow cabinet under ultraviolet light for 1 hour from the initial setting and then placed separately in polypropylene tubes with $5 \mathrm{~mL}$ incomplete DMEM medium and stored refrigerated for 72 hours before the experiment. The remaining material was discarded; the extracts were filtered, and 1:10 and 1:100 serial dilutions were prepared from the initial extracts (1:1). The 1:1, 1:10, and 1:100 solutions were stored refrigerated until use.

\section{3-[4,5-dimethylthiazol-2-y]]-2,5-diphenyl tetrazolium Bromide Assay: Cell Viability}

$1 \times 10^{5}$ cells/well were plated in a 96-well plate, preincubated for 12 hours in serum-free media, and then treated with different MTA and Biodentine extracts for 24 and 48 hours. A tetrazolium dye 3-(4,5dimethylthiazol-2-yl)-2,5-diphenyl tetrazolium bromide $(0.5 \mathrm{mg} / \mathrm{mL}$, Sigma M-5655 MTT Colorimetric Assay; Sigma-Aldrich, St Louis, MO) was added to each well and incubated at $37^{\circ} \mathrm{C}$ for 3 hours in the dark. The absorbance in each well was determined at $570 \mathrm{~nm}$ using a microplate reader ( $\mu$ Quanti; Bio-tek Instruments, Inc, Winooski, VT). Cell viability in each group was expressed as a percentage and compared with the control group (culture medium). Data were analyzed using 1-way analysis of variance followed by the Bonferroni post test $(\alpha=0.05)$.

\section{Quantitative Reverse-transcriptase Polymerase Chain Reaction: Expression of Mineralization-related Genes}

$S P P 1, I B S P, D S P P, A L P L, D M P 1$, and RUNX2 messenger RNA levels were assayed by quantitative reverse-transcriptase chain reaction using fluorescent markers. After stimulation with MTA and Biodentine extracts, cells were harvested for the extraction of total RNA by the column method using thiocyanate guanidine (RNEasy; Qiagen Inc, Valencia, CA) according to the manufacturer's instructions. Total RNA was estimated using $2 \mu \mathrm{L}$ of each sample with a Nanodrop spectrophotometer (Thermo Scientific, Wilmington, DE).

Next, reverse transcription was performed for complementary DNA synthesis followed by polymerase chain reaction. Primers and probes for SPP1 (Hs00959010_m1), IBSP (Hs00173720_m1), DSPP (Hs00171962_m1), ALPL (Hs01029144_m1), RUNX2 (Hs00231692_m1), and DMP1 (Hs01009391_g1) were obtained commercially and are proprietary; thus, sequences are not available (TaqMan Gene Expression Assay; Applied Biosystems, Foster City, CA). Glyceraldehyde-3-phosphate dehydrogenase (Hs 02758991_g1) and beta-actin (Hs 01060665_g1) were used as reference genes. Quantitative reverse-transcriptase polymerase chain reactions were performed in duplicate using a StepOne Plus real-time PCR system (Applied Biosystems).

Amplification was performed under the following conditions: activation of AmpliTaq Gold Enzyme (Applied Biosystems) at $95^{\circ} \mathrm{C}$ for $2 \mathrm{mi}-$ nutes followed by 40 cycles at $95^{\circ} \mathrm{C}$ for 1 second for DNA denaturation and $60^{\circ} \mathrm{C}$ for 20 seconds for primer annealing and polymerization. The results were analyzed based on cycle threshold values. Distilled deionized water was used as a negative control of each primer probe pair. Relative expression was calculated based on the equation $2^{-\Delta \Delta \mathrm{ct}}$.

Relative messenger RNA expression was compared among the groups using 1-way analysis of variance followed by the Tukey test $(\alpha=0.05)$

\section{Acquisition of Slides after In Vivo Pulpotomy}

The slides used in this section were obtained from the specimen database at the Children's Clinic Department, School of Dentistry of Ribeirão Preto, University of São Paulo, Brazil, in accordance with ISO 7405:2008 (22). This study was approved by the Institutional Animal Research Ethics Committee (process \#2013.1.643.58.5).

In total, 87 specimens of the upper second and third premolars and the lower second, third, and fourth premolars from four 12month-old male and female Beagle dogs treated with MTA $(n=35)$ and Biodentine $(n=52)$ were evaluated 120 days after pulpotomy.

\section{Immunohistochemistry: In Vivo Osteopontin and Alkaline Phosphatase Staining}

To determine the expression of mineralization-related markers, immunohistochemistry assays for osteopontin and alkaline phosphatase were conducted in the Biodentine $(n=46)$ and MTA $(n=29)$ groups.

The slides were deparaffinized, hydrated in a decreasing ethanol series, and kept in phosphate-buffered saline (PBS). Next, tissue sections were microwaved $(7 \times 12$ seconds at 2 -minute intervals $)$ with sodium citrate buffer $(\mathrm{pH}=6.0)$ for antigen retrieval. After temperature stabilization, the slides were washed with PBS $(3 \times)$ for 5 minutes, and endogenous peroxidase activity was blocked with $3 \%$ hydrogen peroxide for 40 minutes. Slides were further washed with PBS $(3 \times)$ for 5 minutes, and nonspecific binding sites were blocked with 5\%

TABLE 1. Composition and Manufacturer (city and country) of Materials Evaluated

\begin{tabular}{|c|c|c|}
\hline Brand name & Material & Manufacturer \\
\hline Biodentine & $\begin{array}{l}\text { Powder: tricalcium silicate, dicalcium silicate, calcium } \\
\text { carbonate, calcium oxide, iron oxide, zirconium oxide } \\
\text { Liquid: calcium chloride, hydrosoluble polymer }\end{array}$ & Septodont (Saint Maurdes Fosses, France) \\
\hline ProRoot MTA White & $\begin{array}{l}\text { Powder: Portland cement }(75 \%) \text {, bismuth oxide }(20 \%) \text {, calcium } \\
\text { sulfate dihydrate }(5 \%) \text {, tricalcium silicate, dicalcium silicate, } \\
\text { tricalcium aluminate, tetracalcium aluminoferrite } \\
\text { Liquid: water }\end{array}$ & Dentsply Tulsa Dental (Johnson City, TN) \\
\hline
\end{tabular}


A Cell viability - 24 hours

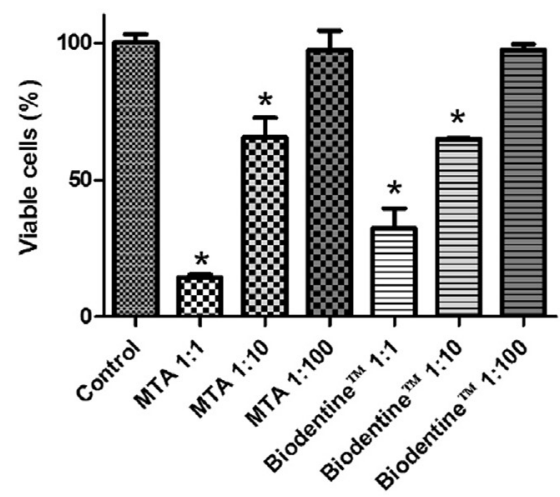

C

SPP1

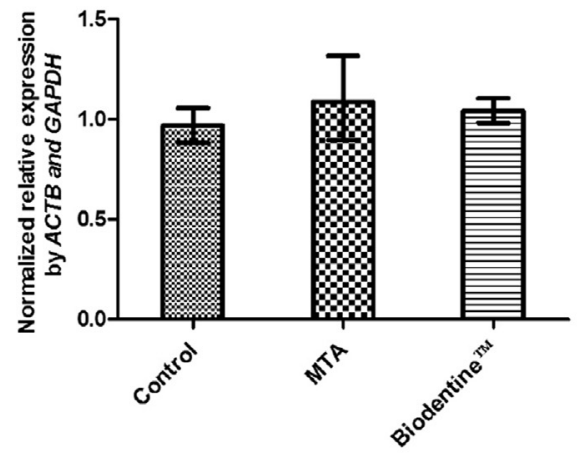

E

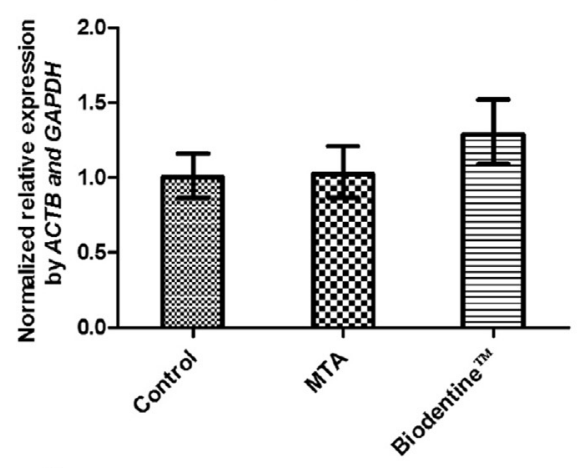

$\mathrm{G}$

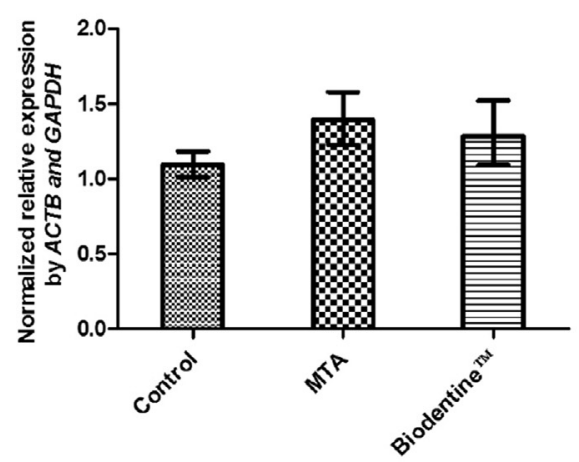

B

Cell viability - 48 hours
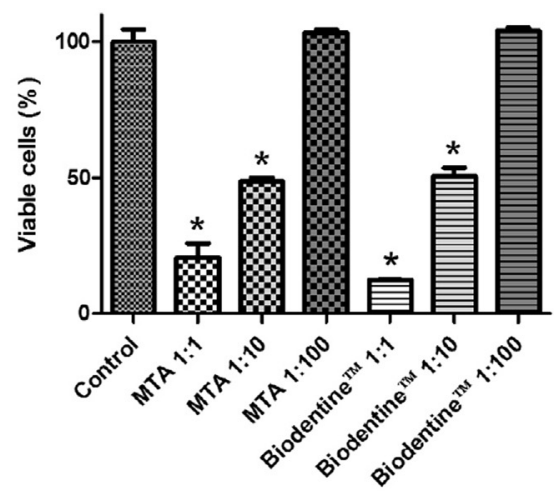

D

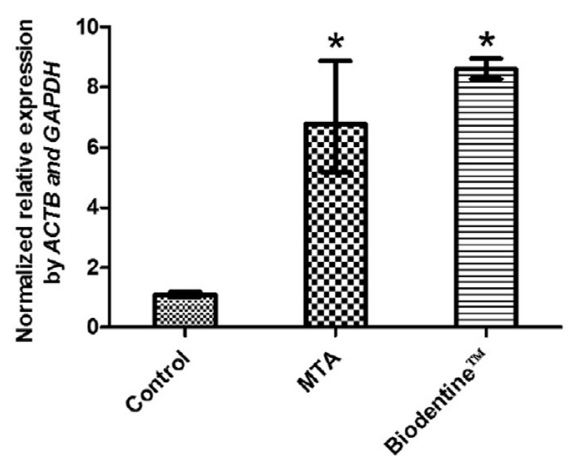

F

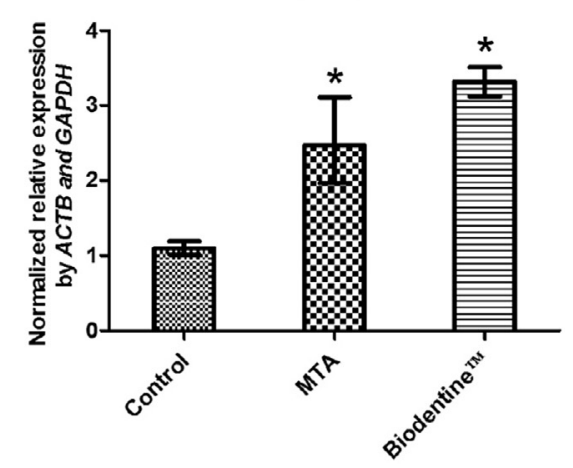

$\mathrm{H}$

RUNX2

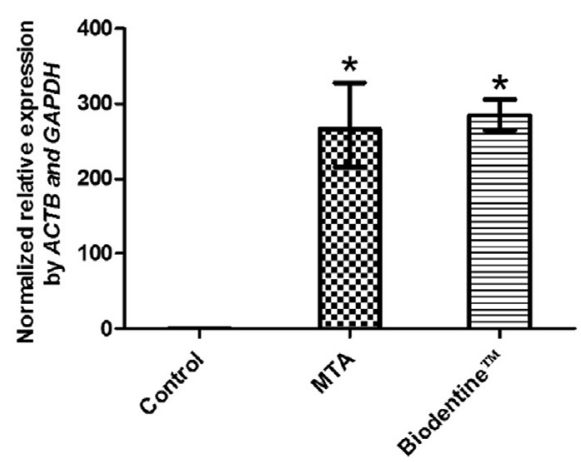

Figure 1. $(A$ and $B)$ The effect of Biodentine and MTA concentration on cell viability in primary culture of dental pulp cells by the 3-(4,5-dimethylthiazol-2-yl)-2,5diphenyl tetrazolium bromide assay after 24 and 48 hours. Gene expression of osteopontin (SPP1), alkaline phosphatase (ALPL), and runt-related transcription factor 2 (RUNX2) compared with the control group after $(C, E$, and $G) 24$ and $(D, F$, and $H) 48$ hours. Significant differences to the control group (cells incubated with culture medium only) are indicated by $*(P<.05)$. Independent experiments $(n=2)$ were done in triplicate. 


\section{Basic Research-Biology}

bovine serum albumin (Sigma-Aldrich) for 60 minutes. The tissues were then incubated with primary antibodies for osteopontin (ab8448, rabbit polyclonal; Abcam, Cambridge, MA) and alkaline phosphatase (ab54778, mouse monoclonal, Abcam) at $4^{\circ} \mathrm{C}$ overnight. Next, slides were washed and incubated with antimouse or antirabbit biotinylated secondary antibodies for 1 hour, washed in PBS, and incubated with streptavidin conjugated with horseradish peroxidase for 20 minutes; $3,3^{\prime}$-diaminobenzidine (Sigma-Aldrich) was used as the enzyme substrate for 5 minutes. The slides were washed with PBS, counterstained with Harris's hematoxylin for 15 seconds, washed with distilled water, dehydrated in increasing ethanol concentrations, and mounted in Entellan (Merck, Darmstadt, Germany). Control slides in which the primary antibody was omitted were used to test the specificity of immunostaining.

Osteopontin and alkaline phosphatase staining in the mineralized tissue bridge and pulp tissue was scored (1: mild, 2: moderate, and 3: intense) at the apical, middle, and cervical root canal thirds. For this analysis, all slides were prepared in the same batch to obtain a standardized staining and were evaluated by an experienced blind examiner. The data were converted into percentage, and groups were compared using the chi-square test $(\alpha=0.05)$.

\section{Indirect Immunofiluorescence: Runt-related Transcription Factor 2 Labeling}

To determine the expression of runt-related transcription factor 2 and its role in the synthesis of mineralization proteins and as an indicator of cell differentiation, indirect immunofluorescence assays were conducted in the Biodentine $(n=6)$ and MTA $(n=6)$ groups.

The slides were prepared as described previously. Next, the slides were washed with PBS $(3 \times)$ for 5 minutes and $1 \mathrm{mg} / \mathrm{mL}$ sodium borohydride solution $(3 \times)$ (Dinâmica Química Contemporânea Ltda, Diadema, SP, Brazil) for 15 minutes. Nonspecific binding sites were blocked with 5\% bovine serum albumin (Sigma-Aldrich) for $60 \mathrm{mi}-$ nutes. Immunolabeling was performed using primary antibody for runt-related transcription factor 2 (ab23981, rabbit polyclonal, Abcam) at $4^{\circ} \mathrm{C}$ overnight. The following day, the slides were washed in PBS $(3 \times)$ and incubated with secondary antibody (antirabbit immunoglobulin $\mathrm{G}$ conjugated with fluorescein) for 1 hour in a dark chamber. The slides were further washed with PBS $(3 \times)$, and the nuclei were stained with $4^{\prime}, 6$-diamidino-2-phenylindole $(0.5 \mu \mathrm{g} / \mathrm{mL}$ ) (Santa Cruz Biotechnology Inc, Dallas, TX) for 5 minutes. Slides were mounted with ProLong Gold Antifade (Molecular Probes Inc, Eugene, $\mathrm{OR})$. The percentage of positively stained cells was determined in the layer adjacent to the newly formed mineralized tissue, the intact odontoblastic layer of pulp tissue, and cells within the central region of this tissue. First, the regions were photographed by fluorescence microscopy at $20 \times$ magnification using Alexa Fluor (Carl Zeiss, Göttingen, Germany) and 4',6-diamidino-2-phenylindole filters. Next, the images were analyzed using ImageJ software (National Institutes of Health, Bethesda, MD) to determine the number of positively stained cells by selecting the "analyze particles" tool; pixel size was set as the average size of previously measured cell nuclei. The total cell count
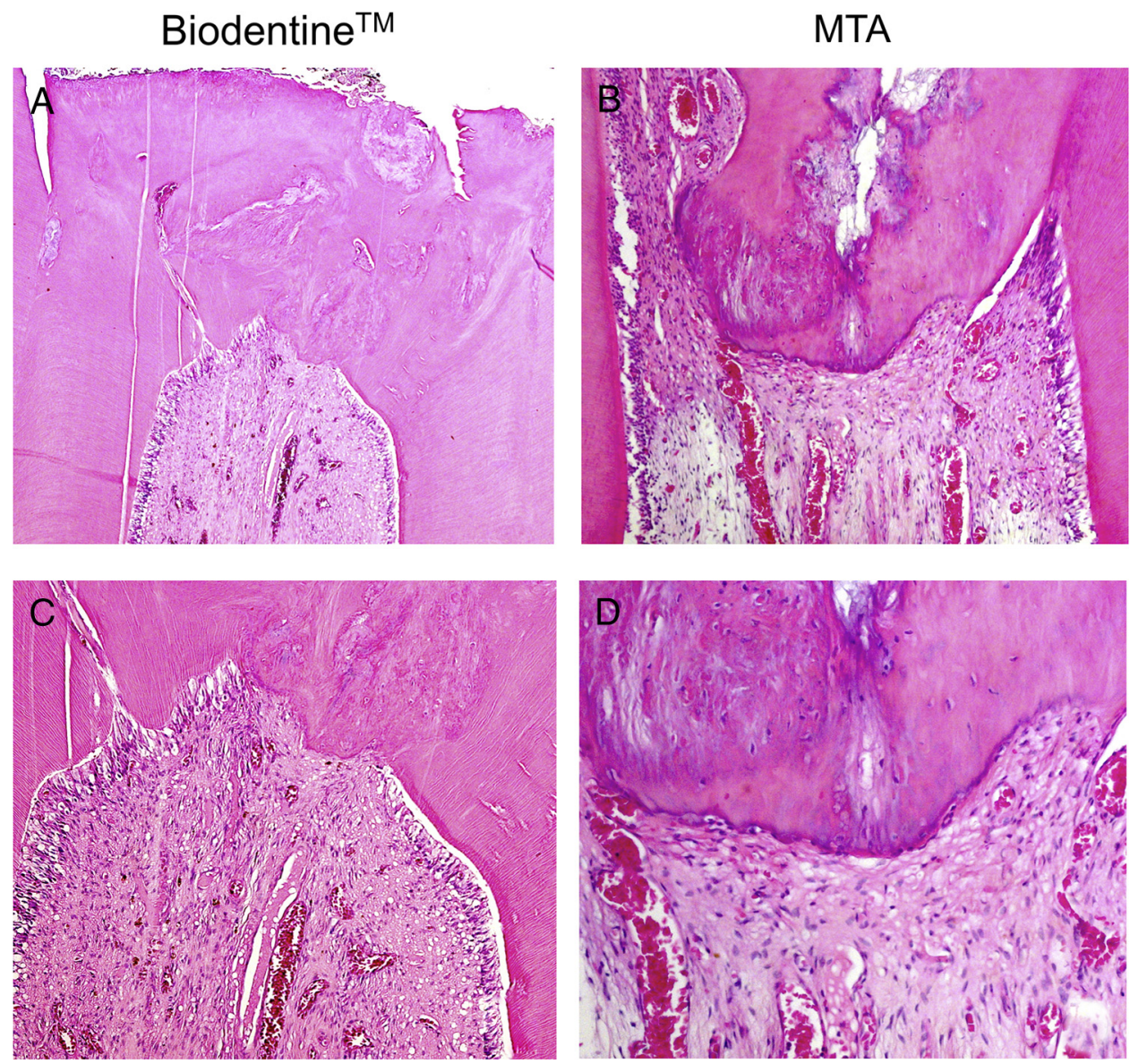

Figure 2. Photomicrographs showing dentin bridge formation within the cervical dental root canal in teeth treated with $(A$ and $C)$ Biodentine and $(B$ and $D)$ MTA. $10 \times(A), 20 \times(B$ and $C)$, and $40 \times(D)$ magnification. 
Basic Research-Biology

and stained cell count were expressed as a percentage, and staining was compared across groups using the Tukey test $(\alpha=0.05)$.

\section{In Vitro}

\section{Results}

Cell viability was significantly lower in 1:1 and 1:10 MTA and Biodentine extracts than in controls at 24 and 48 hours $(P<.05$, Fig. $1 A$ and $B$ ). Conversely, cell viability was not affected by 1:100 dilutions, and no significant differences were found between 1:100 MTA and Biodentine extracts and controls after 24 and 48 hours $(P>.05$, Fig. $1 A$ and $B$ ). Because cell viability was not affected by $1: 100$ dilutions, this concentration was used for the following experiment.

After 24 hours of stimulation, MTA and Biodentine did not modulate SPP1, ALPL, and RUNX2 compared to controls $(P>.05$, Fig. 1C, E, and $G)$. Conversely, MTA and Biodentine induced higher SPP1, ALPL, and RUNX2 gene expression than controls at 48 hours $(P<.05$,
Fig. $1 D, F$, and $H$ ). No IBSP, DSPP, and DMP1 expression was detected in either experimental period.

\section{Mineralization and In Vivo Osteopontin and Alkaline Phosphatase Staining}

Biodentine and MTA induced mineralized tissue formation within the cervical third of the dental root canal pulp (Fig. $2 A-D$ ). We investigated mineralizing proteins that could be involved in this process.

Osteopontin staining of the mineralized tissue bridge and adjacent areas was significantly different between the Biodentine and MTA groups $(P<.0001)$. Mild staining was observed in $29 \%$ of MTA cases, whereas mild, moderate, and intense staining was observed in $26 \%$, $43 \%$, and $22 \%$ of Biodentine cases, respectively (Fig. 3).

Alkaline phosphatase staining of the mineralized tissue bridge was significantly different between filling materials $(P<.0001)$. Mild staining was found in $14 \%$ of MTA cases and $48 \%$ of Biodentine cases (Fig. 4).
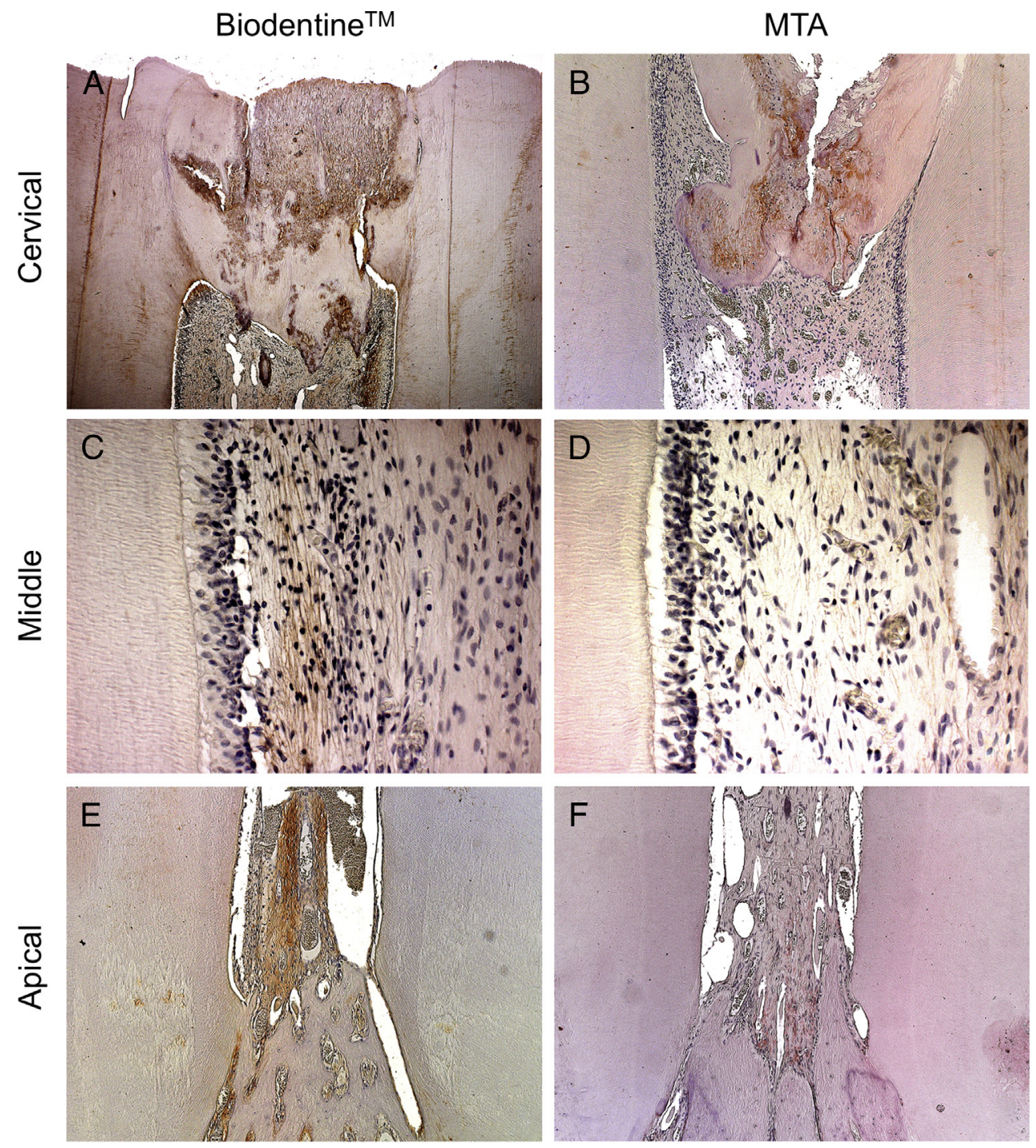

Figure 3. Photomicrographs showing osteopontin staining at cervical, middle, and apical root canal thirds in teeth treated with $(A, C$, and $E)$ Biodentine and $(B, D$, and $F$ ) MTA. Staining of the dentin bridge was detected in more samples and was more intense and extended to the apical third of root canal pulp in Biodentine samples compared with MTA. $5 \times(A), 10 \times(B, E$, and $F)$, and $40 \times(C$ and $D)$ magnification. 


\section{Basic Research-Biology}
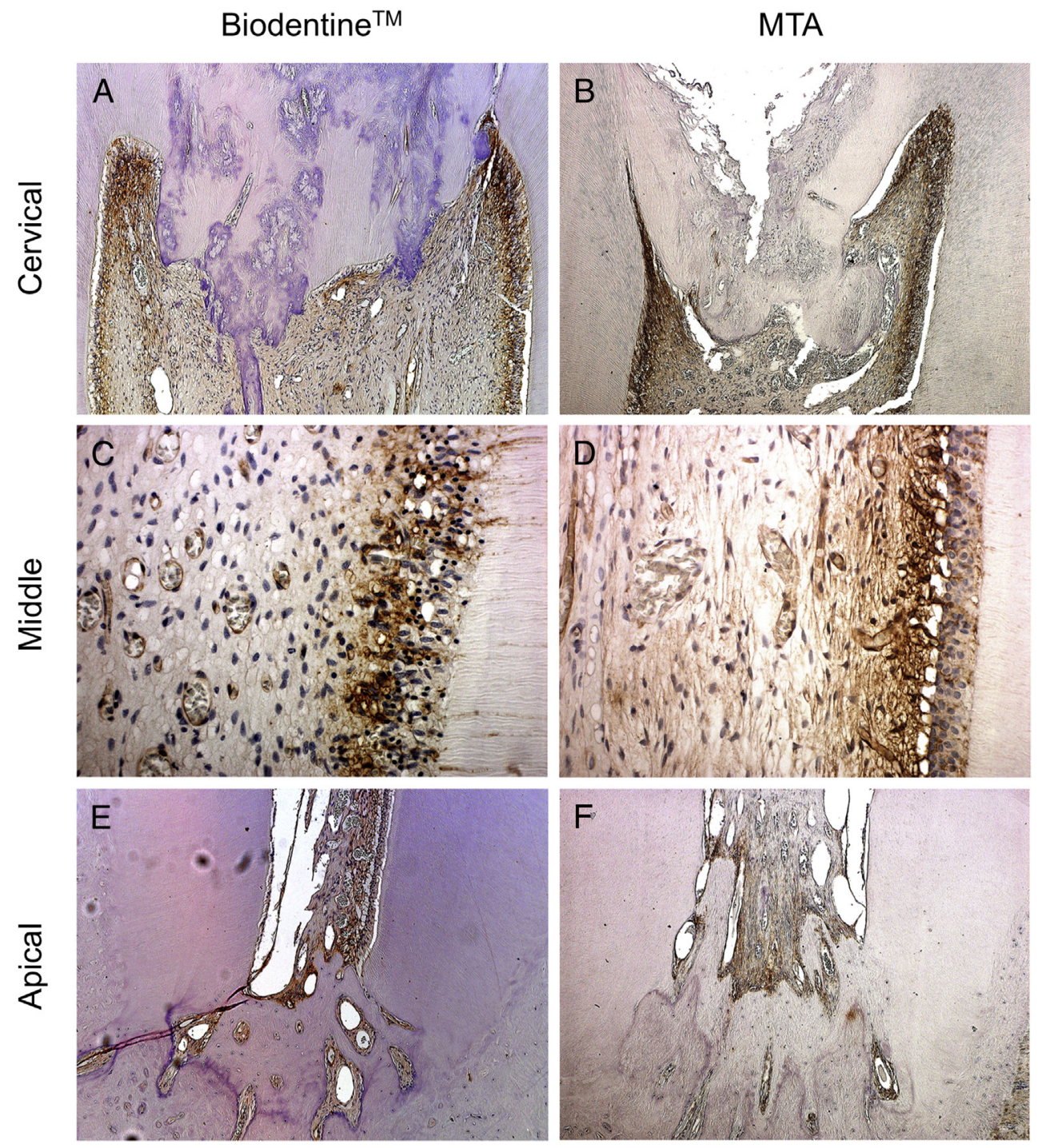

Figure 4. Photomicrographs showing alkaline phosphatase staining at cervical, middle, and apical root canal thirds in teeth treated with $(A, C$, and $E)$ Biodentine and $(B, D$, and $F)$ MTA. Mild staining of the dentin bridge and intense staining of the entire root dental pulp were detected for either Biodentine or MTA. 10 $\times(A, B$, $E$, and $F)$ and $20 \times(C$ and $D)$ magnification.

Osteopontin and alkaline phosphatase staining was also evaluated in pulp tissue at the apical, middle, and cervical root canal thirds. Osteopontin staining was significantly different between MTA and Biodentine $(P<.0001)$ and was concentrated at the cervical third in the 2 groups. For MTA, no staining was observed in $57 \%$ of cases and $29 \%$ of cases were labeled at the cervical and middle thirds, but no staining was observed at the apical third. No staining was observed in $39 \%$ of Biodentine cases, $52 \%$ of cases were stained at the cervical third, and $26 \%$ at the middle and apical thirds (Fig. $3 A-F$ ).

No significant difference in alkaline phosphatase staining of pulp tissue was found between MTA and Biodentine $(P=.2)$. High staining intensity was observed at all root canal thirds in the 2 groups; $100 \%$ of MTA cases were stained at the cervical and middle thirds and $86 \%$ at the apical third, whereas $96 \%$ of Biodentine cases were stained at the cervical third and $91 \%$ at the middle and apical thirds (Fig. $4 A-F$ ).

\section{In Vivo Runt-related Transcription Factor 2 Immunofiluorescence}

No significant difference was found between Biodentine and MTA in the number of positively labeled cells by fluorescence in the central portion of the pulp tissue (Biodentine: $67.3 \%$, MTA: $61.7 \% ; P>.05$, Fig. $5 E-H)$ and the intact odontoblastic layer (Biodentine: $68.7 \%$, MTA: $67 \% ; P>.05$, Fig. $5 I-L)$. Similarly, no significant difference was found in the number of positively stained cells in the region adjacent to newly formed mineralized tissue between Biodentine (87\%) and MTA $(58 \%)(P>.05$, Fig. $5 A-D)$.

\section{Discussion}

Studies comparing the cell viability of Biodentine and MTA have shown the similarity between the 2 materials (23-27). Those results are in agreement with the findings of this study, which showed that Biodentine and MTA had a similar effect on cell viability. In addition to maintaining cell viability, capping materials must be able to induce the formation of mineralized tissue bridges when in contact with pulp tissue in procedures such as pulpotomies and pulp capping treatments. SPP1 and RUNX2 were stimulated in this study in agreement with a previous study (14), indicating that these genes may play an early role in the mineralization process and their longterm maintenance. 


\section{Basic Research-Biology}

\section{MTA}

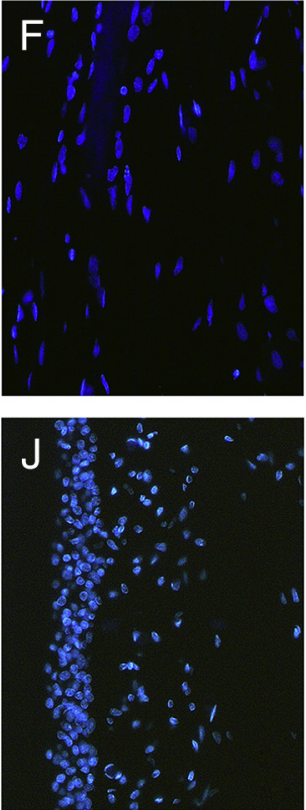

\section{Biodentine $^{\mathrm{TM}}$}
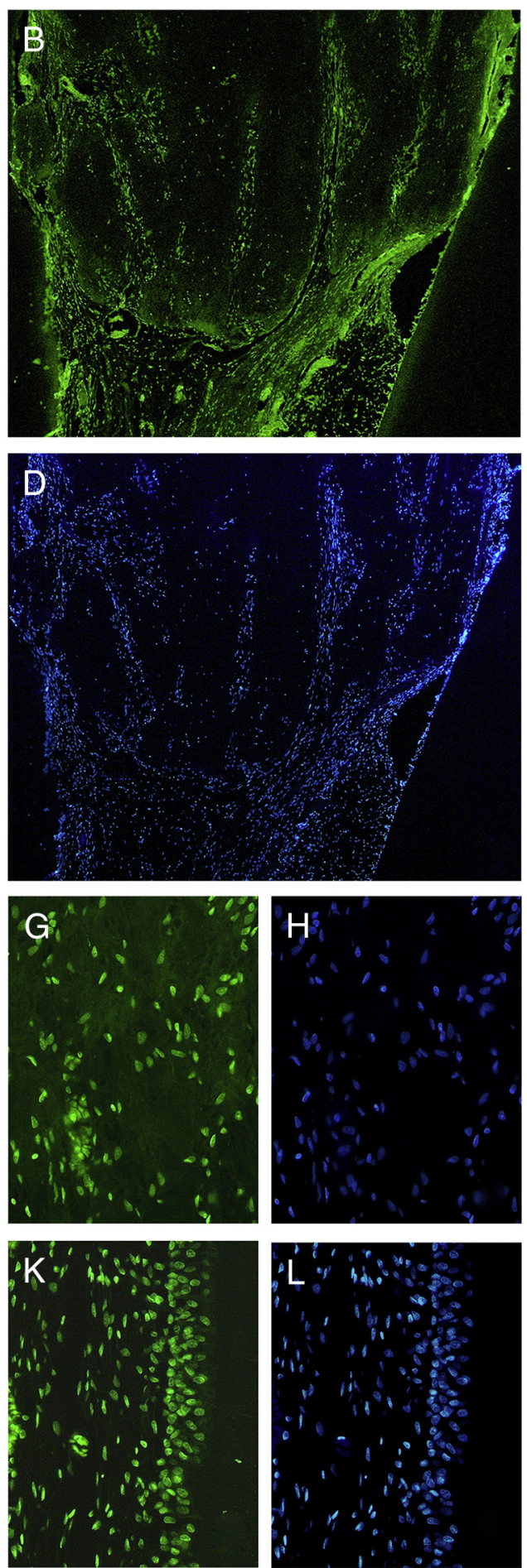

Figure 5. Fluorescence microscopic images showing runt-related transcription factor 2 in teeth treated with $(B, D, G, H, K$, and $L)$ Biodentine and $(A, C, E, F, I$, and $J$ ) MTA using Alexa Fluor and DAPI filters. Similar green fluorescence was observed for the 2 materials in the $(A-D)$ mineralized tissue bridge, $(E-H)$ pulp center, and $(I-L)$ odontoblastic layer. $10 \times(A-D)$ and $40 \times(E-L)$ magnification.

Alkaline phosphatase activity is required for mineral deposition in dentinal collagen sheets (28). In this study, staining was diffuse and extracellular, which is in agreement with other studies that suggested that alkaline phosphatase plays a role in the organism's reaction to injury. Increased $A L P L$ gene expression in vitro has also been observed in other studies after MTA and Biodentine stimulation (29-31). Conversely, decreased alkaline phosphatase expression with simultaneous reduction in enzyme activity has been shown after 


\section{Basic Research-Biology}

11 days of culture, which may be indicative of osteoblast maturation, thus supporting the involvement of this marker in initial mineralization (11).

Increased SPP1 gene expression was also shown in vitro after Biodentine and MTA stimulation in a previous study with no difference between materials (14), which is in line with the current findings. The presence of osteopontin was also investigated by immunohistochemistry in a pulp capping study with calcium hydroxide, MTA, and Biodentine in a rat pulp injury model. The dentin bridge was strongly positive for osteopontin in all samples, whereas adjacent cells showed weak staining in the calcium hydroxide group and more intense staining in the MTA and Biodentine groups (32). Similar results were observed in this study, but osteopontin staining was more intense and spread over a greater number of areas in Biodentine samples. To date, no study had shown such broad osteopontin expression extending to the apical pulp.

The RUNX2 gene coordinates multiple signaling pathways related to osteoblastic differentiation (33), odontoblastic differentiation (34), and tooth development (35). Increased RUNX2 gene expression in vitro was detected in this study and similar findings have been reported previously (14). After longer experimental periods the same pattern of RUNX2 expression induced by both capping materials has also been shown elsewhere (23). Our study detected runt-related transcription factor 2 expression in vivo by immunofluorescence but found no significant difference in expression levels between Biodentine and MTA.

This study showed no significant differences in cell viability between MTA and Biodentine extracts, and both similarly induce SPP1, $A L P L$, and RUNX2 gene expression. In vivo, Biodentine stimulated similar mineralization markers as MTA, but staining was more intense in root dental pulp and dentin bridges. Our findings suggest that Biodentine could be advantageous in the long-term for conservative endodontic treatment to offset the high cost of the material. However, further studies are needed to determine the exact role of each mineralization protein and the signaling pathways that are activated to initiate mineralized tissue formation.

\section{Acknowledgments}

Supported by grants from São Paulo Research Foundation (FAPESP 2010/17611-4 to FWGPS and 2014/25692-5 to LABS) and CAPES Fellowship to MOD.

The authors deny any conflicts of interest related to this study.

\section{References}

1. Camilleri J, Sorrentino F, Damidot D. Investigation of the hydration and bioactivity of radiopacified tricalcium silicate cement, Biodentine and MTA Angelus. Dent Mater 2013;29:580-93.

2. Camilleri J. Investigation of Biodentine as dentine replacement material. J Dent 2013;41:600-10.

3. Laurent P, Camps J, About I. Biodentine(TM) induces TGF-beta1 release from human pulp cells and early dental pulp mineralization. Int Endod J 2012;45:439-48.

4. Wang X, Chang J, Hu S. A study on the sealing ability and antibacterial activity of Ca3Si05/CaCl2 composite cement for dental applications. Dent Mater J 2012;31: 617-22.

5. Koubi G, Colon P, Franquin JC, et al. Clinical evaluation of the performance and safety of a new dentine substitute, Biodentine, in the restoration of posterior teeth - a prospective study. Clin Oral Investig 2013;17:243-9.

6. Yoshino P, Nishiyama CK, Modena KC, et al. In vitro cytotoxicity of white MTA, MTA Fillapex $(\mathrm{R})$ and Portland cement on human periodontal ligament fibroblasts. Braz Dent J 2013;24:111-6.
7. De Rossi A, Silva LA, Gaton-Hernandez P, et al. Comparison of pulpal responses to pulpotomy and pulp capping with biodentine and mineral trioxide aggregate in dogs. J Endod 2014; 40:1362-9.

8. Bachoo IK, Seymour D, Brunton P. Clinical case reports using a novel calciumbased cement. Br Dent J 2013;214:61-4.

9. Villat C, Grosgogeat B, Seux D, Farge P. Conservative approach of a symptomatic carious immature permanent tooth using a tricalcium silicate cement (Biodentine): a case report. Restor Dent Endod 2013;38:258-62.

10. Bhat SS, Hegde SK, Adhikari F, Bhat VS. Direct pulp capping in an immature incisor using a new bioactive material. Contemp Clin Dent 2014;5:393-6.

11. Zanini M, Sautier JM, Berdal A, Simon S. Biodentine induces immortalized murine pulp cell differentiation into odontoblast-like cells and stimulates biomineralization. J Endod 2012;38:1220-6.

12. Nowicka A, Lipski M, Parafiniuk M, et al. Response of human dental pulp capped with biodentine and mineral trioxide aggregate. J Endod 2013;39:743-7.

13. Lee SK, Lee CY, Kook YA, Kim EC. Mechanical stress promotes odontoblastic differentiation via the heme oxygenase-1 pathway in human dental pulp cell line. Life Sci 2010;86:107-14.

14. Chang SW, Lee SY, Ann HJ, et al. Effects of calcium silicate endodontic cements on biocompatibility and mineralization-inducing potentials in human dental pulp cells. J Endod 2014;40:1194-200.

15. Goldberg M, Septier D, Lecolle S, et al. Dental mineralization. Int J Dev Biol 1995;39: 93-110.

16. Chen S, Rani S, Wu Y, et al. Differential regulation of dentin sialophosphoprotein expression by Runx2 during odontoblast cytodifferentiation. J Biol Chem 2005; 280:29717-27.

17. Schouten C, van den Beucken JJ, de Jonge LT, et al. The effect of alkaline phosphatase coated onto titanium alloys on bone responses in rats. Biomaterials 2009;30: 6407-17.

18. Denhardt D'T, Noda M, O'Regan AW, et al. Osteopontin as a means to cope with environmental insults: regulation of inflammation, tissue remodeling, and cell survival. J Clin Invest 2001;107:1055-61.

19. Gaikwad JS, Cavender A, D'Souza RN. Identification of tooth-specific downstream targets of Runx2. Gene 2001;279:91-7.

20. Fisher S, Franz-Odendaal T. Evolution of the bone gene regulatory network. Curr Opin Genet Dev 2012;22:390-7.

21. ISO. Biological evaluation of medical devices. IPart 5: tests for in vitro cytotoxicity. Switzerland: ISO; 2009.

22. ISO. Evaluation of biocompatibility of medical devices used in dentistry. Switzerland: ISO; 2008.

23. Perard M, Le Clerc J, Watrin T, et al. Spheroid model study comparing the biocompatibility of Biodentine and MTA. J Mater Sci Mater Med 2013;24:1527-34.

24. Zhou HM, Shen Y, Wang ZJ, et al. In vitro cytotoxicity evaluation of a novel root repair material. J Endod 2013;39:478-83.

25. Attik GN, Villat C, Hallay F, et al. In vitro biocompatibility of a dentine substitute cement on human MG63 osteoblasts cells: Biodentine ${ }^{\text {TM }}$ versus MTA $\left({ }^{\circledR}\right)$. Int Endod J 2014; $47: 1133-41$.

26. Luo Z, Li D, Kohli MR, et al. Effect of Biodentine on the proliferation, migration and adhesion of human dental pulp stem cells. J Dent 2014;42:490-7.

27. Poggio C, Ceci M, Beltrami R, et al. Biocompatibility of a new pulp capping cement. Ann Stomatol (Roma) 2014;5:69-76.

28. Beertsen W, van den Bos T. Alkaline phosphatase induces the mineralization of sheets of collagen implanted subcutaneously in the rat. J Clin Invest 1992;89:1974-80.

29. Peng W, Liu W, Zhai W, et al. Effect of tricalcium silicate on the proliferation and odontogenic differentiation of human dental pulp cells. J Endod 2011;37:1240-6.

30. Zhao X, He W, Song Z, et al. Mineral trioxide aggregate promotes odontoblastic differentiation via mitogen-activated protein kinase pathway in human dental pulp stem cells. Mol Biol Rep 2012;39:215-20.

31. Wang Y, Yan M, Fan Z, et al. Mineral trioxide aggregate enhances the odonto/osteogenic capacity of stem cells from inflammatory dental pulps via NF-kappaB pathway. Oral Dis 2014;20:650-8.

32. Tran XV, Gorin C, Willig C, et al. Effect of a calcium-silicate-based restorative cement on pulp repair. J Dent Res 2012;91:1166-71.

33. Komori T. Regulation of bone development and extracellular matrix protein genes by RUNX2. Cell Tissue Res 2010;339:189-95.

34. Yang F, Xu N, Li D, et al. A feedback loop between RUNX2 and the E3 ligase SMURF1 in regulation of differentiation of human dental pulp stem cells. J Endod 2014;40: 1579-86.

35. D'Souza RN, Aberg T, Gaikwad J, et al. Cbfa1 is required for epithelial-mesenchymal interactions regulating tooth development in mice. Development 1999;126: 2911-20. 